\title{
Evidence for a single hydrogen molecule connected by an atomic chain
}

Kiguchi, M.; Stadler, Robert; Bækgaard, Iben Sig Buur; Djukic, D.; van Ruitenbeek, J.M.

Published in:

Physical Review Letters

Link to article, DOI:

10.1103/PhysRevLett.98.146802

Publication date:

2007

Document Version

Publisher's PDF, also known as Version of record

Link back to DTU Orbit

Citation (APA):

Kiguchi, M., Stadler, R., Bækgaard, I. S. B., Djukic, D., \& van Ruitenbeek, J. M. (2007). Evidence for a single hydrogen molecule connected by an atomic chain. Physical Review Letters, 98(14), 146802.

https://doi.org/10.1103/PhysRevLett.98.146802

\section{General rights}

Copyright and moral rights for the publications made accessible in the public portal are retained by the authors and/or other copyright owners and it is a condition of accessing publications that users recognise and abide by the legal requirements associated with these rights.

- Users may download and print one copy of any publication from the public portal for the purpose of private study or research.

- You may not further distribute the material or use it for any profit-making activity or commercial gain

- You may freely distribute the URL identifying the publication in the public portal 


\title{
Evidence for a Single Hydrogen Molecule Connected by an Atomic Chain
}

\author{
M. Kiguchi, ${ }^{1, *}$ R. Stadler, ${ }^{2}$ I. S. Kristensen, ${ }^{2}$ D. Djukic, ${ }^{1}$ and J. M. van Ruitenbeek ${ }^{1, \dagger}$ \\ ${ }^{1}$ Kamerlingh Onnes Laboratorium, Universiteit Leiden, P.O. Box 9504, NL-2300 RA Leiden, The Netherlands \\ ${ }^{2}$ Center for Atomic-scale Materials Physics, Department of Physics, NanoDTU, Technical University of Denmark, \\ DK-2800 Kgs. Lyngby, Denmark
}

(Received 28 December 2006; published 4 April 2007)

\begin{abstract}
Stable, single-molecule conducting-bridge configurations are typically identified from peak structures in a conductance histogram. In previous work on $\mathrm{Pt}$ with $\mathrm{H}_{2}$ at cryogenic temperatures it has been shown that a peak near $1 G_{0}$ identifies a single-molecule $\mathrm{Pt}-\mathrm{H}_{2}-\mathrm{Pt}$ bridge. The histogram shows an additional structure with lower conductance that has not been identified. Here, we show that it is likely due to a hydrogen decorated $\mathrm{Pt}$ chain in contact with the $\mathrm{H}_{2}$ molecular bridge.
\end{abstract}

DOI: 10.1103/PhysRevLett.98.146802

The interest in chains of single metal atoms bridging between two electrodes is largely due to their unique properties as ideal one-dimensional systems [1]. For clean metals, only $\mathrm{Au}, \mathrm{Pt}$, and $\mathrm{Ir}$ form atomic chains [2,3]. However, atomic or molecular adsorption on metal surfaces can widen this scope. Recently, $2 \mathrm{~nm}$ long Ag atomic chains have been created in the presence of oxygen at ultralow temperature, while clean $\mathrm{Ag}$ only forms short chains [4]. Atomic chains have been imaged by transmission electron microscope for the noble metals $\mathrm{Cu}, \mathrm{Ag}$, and Au [5-8].

In the first experiments contacting molecules by $\mathrm{Pt}$ atomic leads it was shown that a single hydrogen molecule $\mathrm{H}_{2}$ can be contacted and there appeared to be no indication for atomic chain formation $[9,10]$. By use of point contact spectroscopy and shot noise measurements, the system was characterized in detail and close agreement with atomistic model calculations was obtained $[9,10]$. The $\mathrm{Pt}-\mathrm{H}_{2}-\mathrm{Pt}$ junction was first identified by its conductance. It shows up as a recurring plateau in the conductance when controllably breaking a contact, and in a histogram of conductance values collected for many such breakings it gives rise to a sharp peak near $1 G_{0}$, where $1 G_{0}=2 e^{2} / h$ is the conductance quantum. This main peak at $1 G_{0}$ for the $\mathrm{Pt} / \mathrm{H}_{2}$ system is therefore well understood. However, there is more structure in the conductance histogram for the $\mathrm{Pt} / \mathrm{H}_{2}$ junctions, which has not been explained. In particular, a strong peak is found at about $0.1-0.2 G_{0}$, suggesting that other configurations of hydrogen between Pt leads may be formed. In the present study we focus on those structures, having a conductance below $1 G_{0}$, and we present evidence that they can be attributed to the formation of a hydrogen decorated $\mathrm{Pt}$ atomic chain that forms one of the leads contacting a hydrogen molecule.

The measurements have been performed using the mechanically controllable break junction technique (see Ref. [11] for a detailed description). Once under vacuum and cooled to $4.2 \mathrm{~K}$ a fine Pt wire was broken. Atomicsized contacts between the wire ends can be formed using a
PACS numbers: 73.63.Rt, 63.22.+m, 73.23. $-\mathrm{b}, 85.65 .+\mathrm{h}$

piezoelement for fine adjustment. $\mathrm{H}_{2}$ was admitted via a capillary. dc two-point voltage-biased conductance measurements were performed by applying a voltage in the range from 10 to $150 \mathrm{mV}$. Every statistical data set was built from a large number (over 3000) of individual digitized conductance traces. ac voltage bias conductance measurements were performed using a standard lock-in technique. The conductance was recorded for fixed contact configuration using an ac modulation of $1 \mathrm{mV}$ amplitude and a frequency of $7.777 \mathrm{kHz}$, while slowly ramping the dc bias between -100 and $+100 \mathrm{mV}$.

Figures 1(a) and 1(b) show typical conductance traces for clean $\mathrm{Pt}$ and for $\mathrm{Pt}$ after admitting $\mathrm{H}_{2}$. After admitting $\mathrm{H}_{2}$, plateau near $1 G_{0}$ are frequently observed and the corresponding histogram [Fig. 1(c)] shows a sharp feature near $1 G_{0}$. The plateau near $1 G_{0}$ and the corresponding peak in the histogram originate from single-molecule $\mathrm{Pt}-\mathrm{H}_{2}-\mathrm{Pt}$ contacts, as shown by previous studies $[9,10]$. In addition to the $1 G_{0}$ feature, the histogram shows a peak near $0.2 G_{0}$ on top of a low-conductance tail. Looking at the individual traces, the conductance decreases by small steps after the appearance of a plateau near $1 G_{0}\left(0.8-1.2 G_{0}\right)$ for $92 \%$ of the traces, and the conductance decreases below
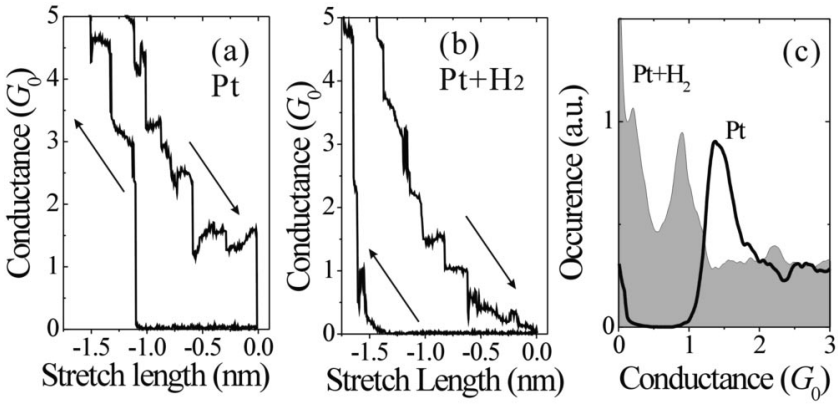

FIG. 1. Breaking and return traces for clean Pt (a), and for Pt in a $\mathrm{H}_{2}$ atmosphere (b). Many of such curves are collected into conductance histograms as shown in (c) for clean Pt (black curve) and $\mathrm{Pt}$ in $\mathrm{H}_{2}$ (filled graph). 
$1 G_{0}$ without appearance of a plateau near $1 G_{0}$ for $8 \%$ of the traces. The sudden drop of the conductance from $1 G_{0}$ to $0 G_{0}$ is a rare event (below 1\%). This suggests that a structure having a conductance below $1 G_{0}$ is formed by further stretching the original single-molecule $\mathrm{Pt}-\mathrm{H}_{2}-\mathrm{Pt}$ contact with a conductance of $1 G_{0}$. The conductance trace in Fig. 1(b) shows that the structure can be stretched to quite long lengths [ $>0.5 \mathrm{~nm}$ in Fig. 1(b)], which suggests the formation of an atomic chain.

In order to investigate the chain formation, we measure the length histogram of the last plateau and the return length distribution. Figure 2(a) shows the length histogram for the final conductance plateau for the $\mathrm{Pt} / \mathrm{H}_{2}$ contacts (filled gray distribution), and this is compared to a length histogram for clean $\mathrm{Pt}$. The length for $\mathrm{Pt} / \mathrm{H}_{2}$ is taken here as the distance between the points at which the conductance drops below 1.3 and $0.1 G_{0}$, respectively, while for clean $\mathrm{Pt}$ the boundaries are 2.5 and $1.0 G_{0}$. The former boundaries are set such as to capture the length of the $1 G_{0}$ plateau plus the subsequent structures that give rise to the peak around $0.2 G_{0}$ in the conductance histogram. It is striking that the $\mathrm{Pt} / \mathrm{H}_{2}$ contact can be stretched as long as $0.8 \mathrm{~nm}$. A sequence of peaks is observed in the $\mathrm{Pt} / \mathrm{H}_{2}$ length histogram of Fig. 2(a), indicating the repeated occurrence of certain stable chain configurations that we identify as (A), (B), and (C). The distance between the peaks is $0.27 \pm 0.01 \mathrm{~nm}$, which is slightly larger than the Pt-Pt distance of a clean Pt atomic chain $(0.23 \mathrm{~nm})$ [3].

The inset of Fig. 2(a) shows the average return lengths as a function of chain length. This is the distance over which the two electrodes need to be moved back after the junction breaks in order to reestablish contact, averaged over many break cycles. Apart from an offset of $0.3 \mathrm{~nm}$ due to the elastic response of the banks [3], the relation is approximately proportional, suggesting that a fragile structure is formed with a length corresponding to that of the last
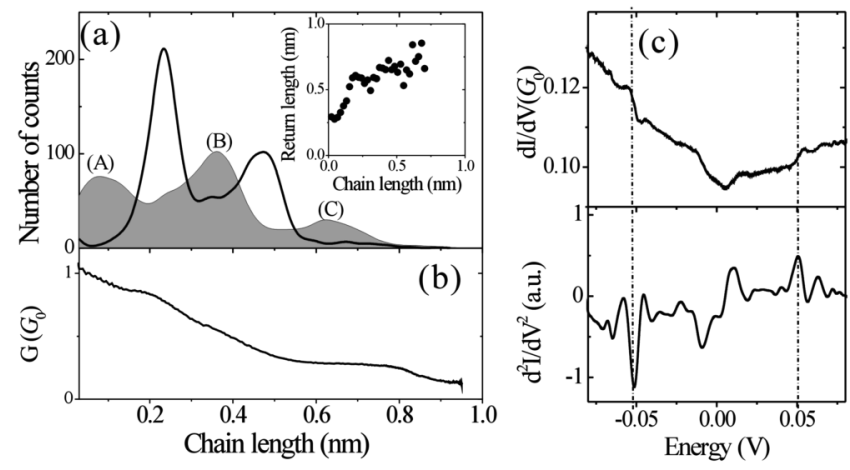

FIG. 2. (a) Length histogram for clean Pt (black curve) and Pt in $\mathrm{H}_{2}$ (filled graph). The start and stop values between which the lengths were measured were taken as $(2.5,1.0)$ for $\mathrm{Pt}$ and $(1.3,0.1)$ for $\mathrm{Pt} / \mathrm{H}_{2}$, in units of $G_{0}$. Inset: Average return lengths as a function of chain length. (b) Average conductance as a function of chain length for $\mathrm{Pt} / \mathrm{H}_{2}$. (c) Differential conductance (top) and its derivative (bottom) for a $\mathrm{Pt} / \mathrm{H}_{2}$ contact taken at a conductance of $0.1 G_{0}$. plateau, which is unable to support itself when it breaks and collapses onto the banks on either side.

We further test this interpretation by analyzing the stretch length dependence of the conductance and by point contact spectroscopy. Figure 2(b) shows the average conductance for $\mathrm{Pt} / \mathrm{H}_{2}$ junctions as a function of the chain length. The curve is obtained by adding all measured conductance traces from the start value $\left(1.3 G_{0}\right)$ onward, and dividing at each length by the number of traces included at that point. The mean conductance decreases rapidly as the chain becomes longer. Although the conductance for a pure Pt chain also decreases with length [12], its conductance stays well above $1 G_{0}$. This fact, combined with the larger peak distance in the length histogram, indicates that the structure with lower conductance is probably not a clean Pt atomic chain, and may be due to a hydrogen decorated atomic chain. The average conductances of structures (A), (B), and (C) are 0.96, 0.56, and $0.28 G_{0}$, respectively. Since the conductance of structure $(\mathrm{A})$ is close to $1 G_{0}$, we identify it with the single-molecule $\mathrm{Pt}-\mathrm{H}_{2}-\mathrm{Pt}$ contacts that have been studied previously $[9,10]$. We discuss the new structures (B) and (C) that arise by further stretching of the $\mathrm{Pt}-\mathrm{H}_{2}-\mathrm{Pt}$ junction in the following. Note that a stable level near $0.2-0.3 G_{0}$ is observed at $0.6-0.8 \mathrm{~nm}$ in length in Fig. 2(b). The slow length dependence gives rise to a high number of counts in a conductance histogram, which explains the peak at $0.2 G_{0}$ in Fig. 1(c). Apart from this stable structure that we have labeled $(C)$ the length histogram points at an intermediate structure (B), for which the conductance varies more strongly with stretching.

Figure 2(c) shows an example of the differential conductance and its derivative for a $\mathrm{Pt} / \mathrm{H}_{2}$ contact taken at a conductance of $0.1 G_{0}$. Clear symmetric peaks are observed at $51 \mathrm{meV}$ in the second derivative, $d^{2} I / d V^{2}$. The peaks are commonly observed near $\sim 57 \pm 4 \mathrm{meV}$ for contacts having conductances in the range $0.6-0.1 G_{0}$. The energy of $57 \mathrm{meV}$ agrees with the energy of the transverse translation mode of the molecule in the Pt-H-H-Pt configuration [10]. This close agreement suggests that a hydrogen molecule is still bridging the junction after stretching it beyond the $1 G_{0}$ plateau. Note that, in most measurements peaks are observed in the first derivative instead of the second derivative, where the analysis outlined in Ref. [13] was used to identify characteristic frequencies.

Based on the experiments presented above we arrive at the following chain formation model for Pt atomic contacts with $\mathrm{H}_{2}$. First, a single hydrogen molecule is adsorbed between Pt electrodes [structure (A)]. Further stretching induces the incorporation of the first Pt atom from the stem part of the electrode into the chain [structure (B)]. Then, the second Pt-atom is incorporated into the chain [structure $(\mathrm{C})$ ], and the atomic chain is formed with a single hydrogen molecule bridging. The larger distances in the length histogram and the low conductance should then be attributed to additional hydrogen decorating the Pt atomic chain. Further support for this chain formation process is 
obtained from density functional theory (DFT) calculations.

Electronic structure calculations were performed using a plane wave implementation of DFT [14] with an energy cutoff of $340 \mathrm{eV}$, where we used ultrasoft pseudopotentials [15], and a PW91 parametrization for the exchange and correlation functional [16]. The transmission functions of the molecular junctions were calculated using a general nonequilibrium Green function formalism for phasecoherent electron transport [17], where both the Green function of the scattering region and the self-energies describing the coupling to the semi-infinite electrodes were evaluated in terms of a basis consisting of maximally localized Wannier functions [18]. In our calculations the supercells for the scattering region are defined by $3 \times 3$ atoms in the surface plane and contain three to four surface layers on each side of the molecule. We used a $4 \times 4$ grid for the $k$-point integration in order to obtain well converged results for the conductance [19].

Based upon the distances and conductances found in the experiment presented above, we came to consider the model structures for (B) and (C) illustrated by the insets in Fig. 3. The figure shows calculated transmission functions for structures (B) and (C) as a function of energy. The transmission function for structure (A), a hydrogen bridge with no additional hydrogen having a conductance of $1 G_{0}$ [20], is lowered when going from (A) to (C). The conductances are found as 0.46 and $0.15 G_{0}$ for structures (B) and (C), respectively, which is in reasonable agreement with the experimental values of $\sim 0.6$ and $\sim 0.3 G_{0}$. The theoretical values have been obtained after optimizing the distance between the contacts by total energy minimization and the optimal length of a $\mathrm{Pt}$ wire segment decorated with additional hydrogen was found to be $0.272 \mathrm{~nm}$ which agrees with the experimental results.

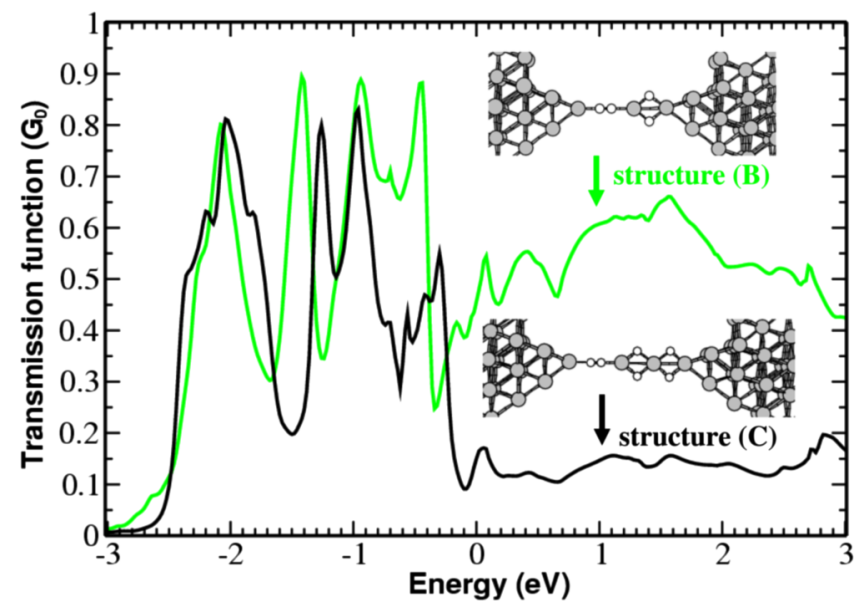

FIG. 3 (color online). Transmission functions for the proposed structures (B) solid gray (green) lines and (C) black lines, respectively, as calculated from DFT for a setup with bulk electrodes and a (111) surface with a pyramid of Pt atoms on top. The zero point of the $x$ axis is the Fermi energy. The explicit geometries of the structures are shown as insets.
Our analysis shows that this reduction is due to the additional hydrogen atoms saturating the $s$ orbital and part of the $d$ orbitals between the Pt atoms they are attached to, thereby making them unavailable for electron transport. This is illustrated in Fig. 4(a) that shows the results of calculations for the $\mathrm{H}_{2}$ bridge structure without additional hydrogen. We have simulated the effect hydrogen addition might have by cutting out Pt Wannier functions from the scattering Hamiltonian. The removal of the Pt $s$ orbital for one and two subsequent wire atoms [shown only for the first case in Fig. 4(a)] accounts for the successive reduction of the transmission at around $1 \mathrm{eV}$ and higher above the Fermi level. For the reduction of the transmission directly at $E_{F}$, and therefore the conductance, also a blocking of some of the $d$ orbitals is needed, which is also shown in Fig. 4(a). Although a cutting of the $d$-Wannier functions of the first Pt atom alone does not have a large effect on the conductance and seems to even enhance it, the conduc-
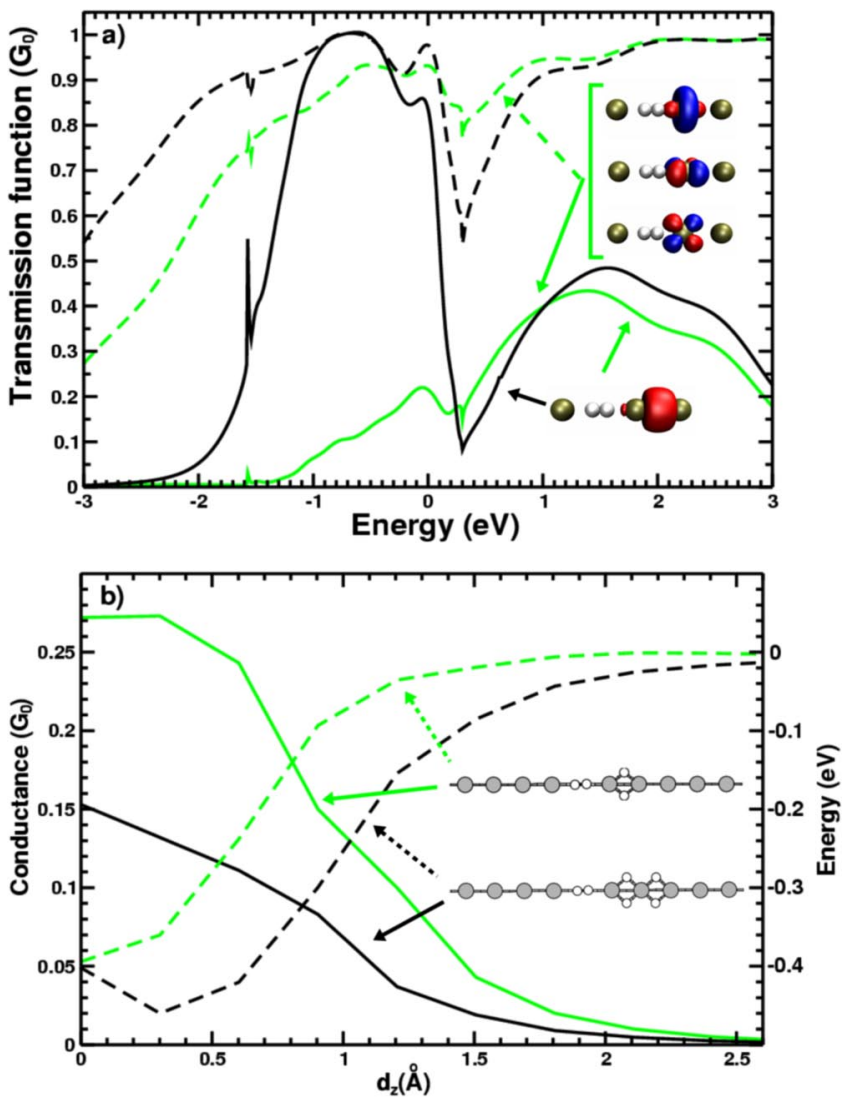

FIG. 4 (color online). (a) Transmission functions obtained from calculations of a single $\mathrm{H}_{2}$ bridge between wires of $\mathrm{Pt}$ atoms with the $s$ orbital (solid black line), the $d$ orbitals [dashed gray (green) line], both the $s$ and $d$ orbitals [solid gray (green) line], and no atomic orbitals (dashed black line) removed from the Hamiltonian. The removed orbitals are also shown superimposed with the atomic positions in the insets. (b) Conductance (solid curves) and binding energy (dashed curves) for the molecule inside the junction for structures similar to (B) [gray (green)] and (C) (black) assumed to have linear atomic wire arrangements as shown in the insets. 
tance is drastically reduced if both the $s$ and $d$ orbitals of the same Pt atom are blocked. We point out that our explanation of the conductance reduction is not in contradiction with the one given by Barnett et al. [21] for the $\mathrm{Au}-\mathrm{H}_{2}-\mathrm{Au}$ system [22] but just offers a different perspective. For structure (B) we also compared the transmission functions of the atomic wire system with that of the more realistic surface calculation in Fig. 3. Qualitatively, the results are similar in terms of the main peak structure illustrating that the analysis is robust with respect to details of the atomic arrangement. In a quantitative comparison, however, the conductance from the surface calculation is higher than for the wire system by approximately a factor of 2.0 .

In Fig. 4(b) we show the dependence of the conductance with increasing the distance between the wire electrodes. In these calculations the positions of all hydrogen atoms and four $\mathrm{Pt}$ atoms on each side have been fully relaxed. The corresponding binding energy of the $\mathrm{H}_{2}$ bridge molecule to the wires is also shown for comparison. Although there is a short plateau in the conductance for structure (B) when stretched beyond the optimal bonding distance, for structure (C) the overall decrease with the length seems to be slower up to a stretching length of $0.1 \mathrm{~nm}$. This trend was also found in the experimental histogram. The shift in optimal distance between (B) and (C) just reflects the increase of the total cell length due to the expansion of a second wire segment.

Let us comment on the path that may lead to the structures discussed above. Since the bridging $\mathrm{H}_{2}$ molecule is very weakly bound to the Pt electrodes, "wire pulling" seems to be unlikely. Structures (B) and (C) can only be formed by a concerted process. Such a process may involve the displacement of $\mathrm{Pt}$ atoms at the surface due to phonons and the formation of intermediate structures with the additional hydrogen adsorbed on the electrodes. The configuration space for covering all possibilities for atomic movements in such a process is too large to be explored by our calculations in detail.

In conclusion, the experimental evidence shows that a $\mathrm{Pt}-\mathrm{H}_{2}-\mathrm{Pt}$ single-molecule junction can be stretched further into forming an atomic wire. We propose a likely structure for this wire in terms of a Pt atomic chain decorated with hydrogen. This interpretation is supported by DFT calculations. While the pathway that brings new atoms into the atomic chain structure remains problematic, we obtain fair agreement in the numbers for the bond distances and the conductances.

We are grateful to K. W. Jacobsen for his support. We acknowledge support from FOM (NOW), Danish National Research Foundation, the Nano-Science Center at the University of Copenhagen, and the DCSC.
*Present address: Division of Chemistry, Graduate School of Science, Hokkaido University, Sapporo 060-0810, Japan.

†Electronic address: ruitenbeek@ physics.leidenuniv.nl

[1] R.E. Peierls, Quantum Theory of Solids (Clarendon, Oxford, 1964).

[2] S. R. Bahn and K.W. Jacobsen, Phys. Rev. Lett. 87, 266101 (2001).

[3] R.H.M. Smit, C. Untiedt, A. I. Yanson, and J.M. van Ruitenbeek, Phys. Rev. Lett. 87, 266102 (2001); A. I. Yanson, G. R. Bollinger, H.E. van den Brom, N. Agrait, and J. M. van Ruitenbeek, Nature (London) 395, 783 (1998).

[4] W.H. A. Thijssen, D. Marjenburgh, R. H. Bremmer, and J. M. van Ruitenbeek, Phys. Rev. Lett. 96, 026806 (2006).

[5] H. Ohnishi, Y. Kondo, and K. Takayanagi, Nature (London) 395, 780 (1998).

[6] V. Rodrigues and D. Ugarte, Phys. Rev. B 63, 073405 (2001).

[7] V. Rodrigues, J. Bettini, A. R. Rocha, L. G. C. Rego, and D. Ugarte, Phys. Rev. B 65, 153402 (2002).

[8] J.C. Gonzalez, V. Rodrigues, J. Bettini, L. G. C. Rego, A. R. Rocha, P.Z. Coura, S. O. Dantas, F. Sato, D. S. Galvao, and D. Ugarte, Phys. Rev. Lett. 93, 126103 (2004).

[9] R. H. M. Smit, Y. Noat, C. Untiedt, N.D. Lang, M. C. van Hemert, and J. M. van Ruitenbeek, Nature (London) 419, 906 (2002).

[10] D. Djukic, K. S. Thygesen, C. Untiedt, R. H. M. Smit, K. W. Jacobsen, and J. M. van Ruitenbeek, Phys. Rev. B 71, 161402(R) (2005); D. Djukic and J.M. van Ruitenbeek, Nano Lett. 6, 789 (2006).

[11] N. Agrait, A. Levy Yeyati, and J. M. van Ruitenbeek, Phys. Rep. 377, 81 (2003).

[12] R.H. M. Smit, C. Untiedt, G. Rubio-Bollinger, R.C. Segers, and J. M. van Ruitenbeek, Phys. Rev. Lett. 91, 076805 (2003).

[13] W. H. A. Thijssen, D. Djukic, A. F. Otte, R. H. Bremmer, and J. M. van Ruitenbeek, Phys. Rev. Lett. 97, 226806 (2006).

[14] B. Hammer, L. B. Hansen, and J. K. Nørskov, Phys. Rev. B 59, 7413 (1999); S. R. Bahn and K. W. Jacobsen, Comput. Sci. Eng. 4, 56 (2002); The DACAPO code can be downloaded at http://www.fysik.dtu.dk/campos.

[15] D. Vanderbilt, Phys. Rev. B 41, R7892 (1990).

[16] J.P. Perdew et al., Phys. Rev. B 46, 6671 (1992).

[17] Y. Meir and N. S. Wingreen, Phys. Rev. Lett. 68, 2512 (1992).

[18] K. S. Thygesen and K. W. Jacobsen, Chem. Phys. 319, 111 (2005).

[19] K. S. Thygesen and K. W. Jacobsen, Phys. Rev. B 72, 033401 (2005).

[20] K. S. Thygesen and K. W. Jacobsen, Phys. Rev. Lett. 94, 036807 (2005).

[21] R. N. Barnett, H. Haekkinen, A. G. Scherbakov, and U. Landman, Nano Lett. 4, 1845 (2004).

[22] S. Csonka, A. Halbritter, and G. Mihaly, Phys. Rev. B 73, 075405 (2006). 